\title{
Em cada lugar um brincar: reflexão evolucionista sobre universalidade e diversidade
}

\author{
Play activity for all places: thinking about the universality \\ and the diversity from an evolutionary perspective
}

\author{
Reginalice de Lima MARQUES \\ Ilka Dias BICHARA
}

\begin{abstract}
Resumo
Pensar sobre a brincadeira infantil como adaptação necessária para a vida, indica um paralelo entre universalidade e diversidade deste fenômeno. Apresentamos aqui evidências apontadas por pesquisas mostrando particularidades observadas nas brincadeiras em contextos específicos. E perguntamos como variações no brincar, relacionadas aos contextos particulares, associam-se ás suas funções no desenvolvimento ontogenético. Nossa reflexão é traçada a partir de uma abordagem evolucionista, relacionando características intrínsecas das brincadeiras com possíveis funções e adaptações filogenéticas e ontogenéticas, na espécie humana. Os resultados descritos nas pesquisas observadas nos indicam evidências do brincar como comportamento funcional e adaptativo para o próprio período da infância, onde as crianças provam seu ambiente e podem aprender e praticar comportamentos adaptáveis a cada ambiente em especial. Além disso, dado o papel fundamental do contexto e cultura na brincadeira, percebemos a possibilidade de investigar como a diversidade do desenvolvimento humano se relaciona com os aspectos universais dos indivíduos.
\end{abstract}

Unitermos: Brincadeira infantil. Diversidade. Perspectiva evolucionista.

\begin{abstract}
Thinking about children's play as a necessary adaptation for human existence, leads us to think about what is universal and what is different about this phenomenon. We present here evidence quoted in various elements of research that provide detail in specific contexts, and we discuss how variation in play is related to functions in ontogenetic development. We follow the evolutionary theories, relating intrinsic characteristics of play to possible functions and phylogenetic and ontogenetic adaptations in the human species. The research examined indicates evidence of play as a functional and adaptive behavior for childhood, where children test their environment and can learn and practice adaptable behavior specific to each environment. Moreover, given the fundamental role of the context and culture in play, we can see that the possibility exists of investigating how the diversity of human development can be linked to the universal aspects of individual development.
\end{abstract}

Uniterms: Children's play. Diversity. Evolutionary theories.

VरV

1 Universidade Federal da Bahia, Faculdade de Filosofia e Ciências Humanas, Programa de Pós-Graduação em Psicologia. Estrada de São Lázaro, 197, 40210-730, Salvador, BA, Brasil. Correspondência para/Correspondenceto: R.L. MARQUES.E-mails: <reginalice@gmail.com>; <reginalicemarques@ufba.br>. 
Quase sempre a observação de crianças em atividades livres revela brincadeiras. Carvalho (1989) chega a dizer que esta parece ser a única atividade desenvolvida por elas. O comportamento de brincar, ou lúdico, apresenta-se como um fenômeno universal na espécie humana. Entretanto, ao observar o brincar humano, pode-se perceber a diversidade existente nesse fenômeno. Tem-se, aí, um aspecto intrigante que merece reflexão: universalidade versus diversidade: crianças de todo o mundo brincam, porém o tipo, a maneira, a companhia, entre outros aspectos, podem variar tanto de um lugar para outro quanto de criança para criança.

Essa diversidade é o tema deste artigo, e, para isso, buscaram-se as evidências apontadas por pesquisas que apresentam as particularidades observadas nas brincadeiras infantis em contextos específicos. Esta reflexão é traçada, ainda, a partir de uma abordagem evolucionista, que associa às características intrínsecas das brincadeiras suas possíveis funções e adaptações tanto em termos filogenéticos quanto ontogenéticos, especialmente na espécie humana. Isso inclui, também, a investigação sobre as variáveis contextuais, ressaltando-as como principal fator responsável pela diversidade de tal comportamento.

O estudo da brincadeira, assim como a busca de explicações sobre a condição humana, através das teorias evolucionistas, como lembrado por Pellegrini, Dupius e Smith (2007), tem sido parte de um desafio vivido ao longo do Século XX. Apenas muito recentemente, a partir dos anos de 1990, tem sido proposta uma Psicologia Evolucionista do Desenvolvimento, definida como a aplicação dos princípios básicos da evolução para explicar o desenvolvimento humano contemporâneo (Bjorklund \& Blasi, 2005). O conhecimento proposto por essa disciplina pode ser também uma base fundamental para discutir vários aspectos acerca da brincadeira, já que se trata de um fenômeno diretamente relacionado ao desenvolvimento humano.

Dentro desse panorama evolucionista, ressalta-se a hipótese ontogenética, que considera a brincadeira um comportamento funcional e adaptativo para o próprio período da infância, em contraposição a crenças predominantes por muito tempo, nas ciências do comportamento e em algumas teorias do desenvolvimento, de que a função primordial da brincadeira seria a de treino de habilidades necessárias à vida adulta.
Se pensarmos na brincadeira como uma adaptação necessária ao tipo de vida dos humanos, que requer grande variabilidade e flexibilidade comportamental, pode-se traçar um paralelo entre o fato de a brincadeira ser ao mesmo tempo universal e diversa. E aí está, principalmente, a relevância da busca de se compreender os fatores que promovem toda variedade que observamos nas brincadeiras. De forma sintética, há então uma pergunta: como as variações, ou diversidades, vistas nas formas de brincar, e relacionadas aos contextos específicos, associam-se às suas funções no desenvolvimento ontogenético?

\section{Dos universais ao diverso no brincar}

Para entender o que pode ser visto como diversidade no fenômeno da brincadeira, é necessário esclarecer quais aspectos, além do próprio fato de brincar, são comuns a todas as crianças. Junto a isso, é necessário expor as principais questões que envolvem as razões, ou funções, pelas quais o comportamento de brincar foi selecionado.

Nesse sentido, qualquer hipótese parte da reflexão sobre o porquê do longo período de imaturidade da espécie ter sido selecionado, já que isso pode representar um perigo potencial na adaptação. Além disso, essa longa espera para reproduzir acompanha o aumento das chances do indivíduo morrer antes de ter descendentes. Uma característica tão custosa quanto essa deve ter também grandes benefícios para ter passado pela peneira da seleção natural. É possível, portanto, que o principal benefício seja o da oportunidade ampliada para aprender as regras e normas sociais de um grupo de indivíduos. Assim, um longo período de imaturidade é necessário também para o aprendizado e domínio da complexidade da cultura humana (Pellegrini et al., 2007).

Muitos aspectos da infância servem como preparação para a idade adulta e foram selecionados no curso da evolução. Nesse caso, o treino para o futuro ocorre principalmente quando o ambiente ou as condições sociais permanecem relativamente estáveis com o passar do tempo. Nessa perspectiva, algumas diferenças relativas ao gênero podem ser bons exemplos dessas adaptações para a vida adulta. Homens e mulheres desenvolvem diferentes tendências comportamentais que 
começam no curso da infância; dessa forma, muitas experiências infantis parecem promover as diferenças de gênero. As diferenciações observadas nas brincadeiras infantis de meninos e de meninas poderiam servir, assim, como uma preparação das crianças para os papéis de gênero quando adultos - considerando o ambiente de adaptação evolucionário (Bjorklund \& Blasi, 2005).

Porém, na infância, observam-se também características que parecem ter sido selecionadas para servir a uma função adaptativa num tempo específico do desenvolvimento e não como preparação para a vida futura. Essas características servem, portanto, para adaptar os indivíduos aos ambientes em que vivem e não para um futuro de forma antecipada - "adaptação ontogenética". As características com função adaptativa não são formas imaturas de adaptação adulta que tomam gradualmente uma forma madura, mas referem-se a comportamentos, estruturas ou mecanismos que têm uma função específica num tempo particular do desenvolvimento e desaparecem quando não são mais necessários - assim como o reflexo perioral (sugar) nos bebês, que é importante para a alimentação no início da vida, mas que desaparece com o crescimento (Bjorklund \& Blasi, 2005).

Segundo Pellegrini e Smith (1998), especialmente na primeira infância, crianças gastam mais tempo explorando o ambiente do que propriamente brincando, particularmente nos primeiros nove meses de vida. Aos 12 meses, brincadeira e exploração ocorrem concomitantemente; aos 18 meses, a brincadeira representa a maior parte das interações da criança com o ambiente. A exploração representa, dessa maneira, um empreendimento para acumular informações, o que pode ser evidenciado, em suas formas iniciais, através da simples manipulação de objetos.

Quando explorando, crianças estão guiadas pela questão"o que isto pode fazer?". Essa questão muda quando a orientação da criança passa a estar mais centrada nela mesma, e a questão transforma-se em "o que eu posso fazer com isto?" (Pellegrini \& Smith, 1998, p.52). Pela exploração, a criança pode conhecer seu ambiente, e esse conhecimento provê as bases para a brincadeira. Microgeneticamente, a exploração também parece preceder a brincadeira: crianças de todas as idades parecem explorar um objeto ou conhecer suas propriedades antes que elas possam brincar com ele.
Como visto, o brincar na primeira infância caracteriza-se pelo brinquedo manipulativo, mas entre 12 e 18 meses também podem ser observadas formas simples do brinquedo simbólico, quando as crianças reordenam esquemas conhecidos por elas em contexto deslocado, ou diferente do real - beber em um copo vazio, por exemplo (Bichara, Lordelo, Carvalho \& Otta, 2009). Com o tempo, esse brinquedo vai refinando-se, e por volta dos três anos de idade, muitas crianças podem fazer transformações simbólicas, ou criar eventos imaginários. Após essa idade, o faz-de-conta envolve a habilidade em papéis sociais sofisticados nas interações com pares, envolvendo atuação completamente complexa em sequências narrativas (Pellegrini \& Smith, 1998).

Assim, observamos a relativa estabilidade da brincadeira entre as diversas populações humanas. Mesmo havendo diferenças cronológicas na aquisição dos tipos de brincadeiras, elas permanecem estáveis inclusive em casos específicos como de crianças prematuras, abandonadas, com retardo mental e com síndrome de down (Bichara et al., 2009).

Pôde-se observar as características universais de funcionalidade e de desenvolvimento ontogenético da brincadeira, ou seja, o percurso de desenvolvimento e formas semelhantes esperadas para as brincadeiras em todas as crianças. Assim, para todas as espécies que brincam, o brincar parece ser uma atividade provedora, entre outras coisas, de oportunidade para desenvolver habilidades motoras, prática do domínio de comportamentos sociais, e aprendizado através da experimentação em situações seguras com o mínimo de consequências (Bjorklund, 1997; Johnson, Christie \& Yawkey, 1999). Porém, a causalidade e os fatores que influenciam as variações nas brincadeiras, em especial os contextos específicos, continuam intrigantes.

As diversas formas de brincadeiras podem ajudar na compreensão da sua associação às variações e às funções do brincar na infância.

\section{A diversidade do brincar: o que se observa?}

Falar em uma hipótese de adaptação ontogenética para a brincadeira implica dizer que o comportamento de brincar representa um componente central do período de imaturidade da espécie humana e encontra-se, especialmente, intrincado ao percurso do 
seu desenvolvimento ontogenético. Especialmente em relação a esses termos, pode-se pensar na diversidade da brincadeira como produto da dinâmica física do organismo, em que o brincar pode ser afetado pelo ambiente - do qual fazem parte as condições físicas, os parceiros sociais, os relacionamentos construídos e os elementos culturais (Bichara et al., 2009).

Assim, de maneira geral, alguns fatores podem ser mais diretamente indicados como possíveis responsáveis pela variabilidade encontrada nas brincadeiras. Esses fatores estão relacionados com a causação da brincadeira. Bichara et al. (2009) afirmam que a mesma dificuldade encontrada para definir esse fenômeno existe também em relação à determinação dos fatores que podem afetar a dinâmica da brincadeira, especialmente no caso da espécie humana. Apesar disso, além de fatores culturais, educacionais e relacionados aos contextos específicos nos quais as crianças brincam, parecem estar envolvidas, ainda, motivações sociais e pessoais.

Para procurar entender além do seu papel no desenvolvimento e na sua funcionalidade, o fenômeno da brincadeira tem sido objeto de inúmeras pesquisas que buscam descrever o brincar em culturas e contextos específicos, associando-o ou não com aspectos do desenvolvimento infantil. Em estudos mais atuais, a importância das interações e do contexto cultural na organização social da brincadeira tem sido salientada por diversos autores (Carvalho \& Pedrosa, 2002; Pontes \& Magalhães, 2003; Santos, 2005; Seixas, 2007). Todos esses tipos de estudos podem ajudar a pensar sobre como as formas variadas de brincar podem demonstrar sua funcionalidade adaptativa na infância, como veremos adiante.

Morais e Otta (2003), em suas observações de brincadeiras entre as crianças da cidade de Ubatuba, litoral do estado de São Paulo, encontraram grande diversidade nas formas de brincar: essas crianças apresentaram um grande elenco de brincadeiras, tanto em forma quanto em conteúdo. As autoras atribuem a variedade encontrada à pouca quantidade de brinquedos estruturados oferecidos às crianças, o que poderia ter favorecido a imaginatividade. Elas indicam que o grande espaço livre disponível para brincar, hábitos de brincadeiras fora da escola e a atitude dos pais em relação ao brincar constituem fatores que podem influenciar a 384 maneira como as crianças brincam.
Sobre esse aspecto, Morais e Otta (2003, p.127) apontam o conceito de "zona lúdica", que, no sentido utilizado pelas autoras, representa o espaço no qual ocorre o brincar, constituído pelos seguintes elementos: o espaço físico propriamente dito, com suas dimensões e conteúdos; o espaço temporal, com o tempo dedicado à brincadeira; o indivíduo, com suas experiências, seus recursos, suas motivações; e as pressões e condições sociais que o cercam. Assim, na zona lúdica podem estar incluídas todas as variáveis que influenciam o brincar das crianças, como, por exemplo: acesso à televisão, disponibilidade de brinquedos, atitudes dos pais e de outros adultos em relação ao brincar, disponibilidade de parceiros (irmãos e amigos), coetâneos ou não, com quem brincar; representações sociais da brincadeira e formas de brincar, bem como a visão e a expectativa que se tem da criança, do adolescente e do adulto numa determinada sociedade.

Neste estudo, zona lúdica aparece como um conceito amplo, um conjunto de fatores associados aos quais podem ser atribuídas as formas de brincar, assim como a grande variedade de brincadeiras (como o que foi visto nas crianças ubatubanas). Outros estudos encontram características peculiares nas brincadeiras infantis pertinentes à cultura específica de determinadas comunidades. Isso também pode ser relacionado ao mesmo conceito de zona lúdica, na medida em que ao se analisarem todos os fatores envolvidos, é possível perceber que tipo de influência pode estar mais diretamente associado a uma forma de brincar característica a um determinado contexto.

Assim, Seixas (2007) destaca, em seu estudo sobre as brincadeiras na Ilha dos Frades (BA), o grande número de brincadeiras associadas ao mar e ao ambiente de praia. Nesse caso, é sugerido que esse resultado fosse, de alguma forma, esperado, uma vez que as próprias características físicas do ambiente, bem como a atividade econômica da região de pesca, são entendidas como aspecto particular que podem influenciar os tipos de brincadeiras desenvolvidos pelas crianças.

Santos (2005) também encontrou especificidades ao relacionar as brincadeiras ao contexto, observando grande quantidade de brincadeiras envolvendo temas sobre caminhões/caminhoneiros. Essa pesquisa foi realizada numa cidade do agreste sergipano, cuja principal atividade econômica é o transporte de carga pesada, 
existindo alta circulação de caminhões e de caminhoneiros.

Esses dois últimos exemplos podem, em especial, ilustrar de que forma fatores específicos ligados ao contexto (levando em conta o conceito apresentado de zona lúdica) emergiriam enquanto influência à alta ocorrência de determinadas formas e conteúdos de brincadeiras em detrimento de outros. Outro ponto saliente é o modo como o mundo adulto (fornecedor de substrato para os conteúdos das brincadeiras) é representado pelas crianças que podem construir suas formas de brincar a partir dele. Entretanto, vale ressaltar, assim como feito por Gosso (2005), que em suas brincadeiras as crianças mostram apenas uma pequena parte do mundo adulto que as cerca, sem, no entanto, reproduzir uma miniatura dessa sociedade.

Gosso (2005) descreve as brincadeiras das crianças pertencentes à tribo indígena Parakanã, da região norte do Brasil, e sugere que as crianças parecem viver numa cultura à parte dos adultos, como uma cultura dentro da outra, mas sem que nenhuma esteja alheia à outra. Quanto às características específicas que influenciam as brincadeiras, ela destaca os reflexos da liberdade vivida por essas crianças. Por exemplo, uma criança pode usar um arco e flecha, produzido pelo próprio pai, para abater pequenos animais de forma segura e protegida. Entretanto, isso não parece incluir uma preocupação direta dirigida ao futuro, e as crianças o fazem, de forma natural, compartilhando com os adultos sua cultura.

Nesse mesmo sentido, discutindo sobre um espaço de compartilhamento da cultura das crianças com a dos adultos, Mekideche (2004, p.150) apresenta a"zanca", que representa um espaço intermediário entre o espaço privado e o público, quer dizer, é o espaço exterior, mais ou menos próximo ao domicílio, livre para a apropriação pelas crianças e vivido por elas em segurança. Trata-se, portanto, do espaço de rua que é compartilhado com pessoas próximas e conhecidas. É nesse espaço que as crianças - de cidades como Argel, Amã ou Cairo - convivem entre si, e seus limites são determinados pela idade e pelo sexo. Conforme a autora, a zanca constitui um espaço educativo de autonomização e socialização da criança e nele as crianças adquirem boa parte de sua aprendizagem sociocognitiva.
Na discussão apresentada por Mekideche (2004), está presente, ainda, como fator que pode afetar diretamente as brincadeiras das crianças, o ambiente, ou seja, a interação da criança com o espaço do qual ela dispõe para realizar sua atividade lúdica. Acerca disso, a autora conclui que as áreas periféricas das cidades oferecem claramente mais pessoas para observar e mais coisas para coletar, manipular e transformar do que nas calçadas cimentadas dos centros das cidades. Nos centros, as crianças, tendo menos possibilidades de atuar sobre os elementos naturais e transformáveis do ambiente físico, voltam-se para os elementos fixos (mobiliário urbano) ou para os corpos dos seus pares, que servem como suporte para as atividades motoras globais.

O ambiente ou o espaço físico tem sido investigado em sua relação mais imediata com os aspectos do comportamento lúdico das crianças. Conforme a revisão sobre brincadeira e desenvolvimento infantil realizada por Johnson et al. (1999), as investigações sobre a influência do ambiente têm-se dirigido para investigar a forma através da qual os arranjos podem estimular ou inibir o aparecimento de certas brincadeiras entre as crianças. Além disso, esses estudos indicam também como as crianças se engajam em brincadeiras conforme o ambiente, que pode ser externo ou interno. O espaço físico, portanto, pode também ser tomado como outro fator específico associado às possíveis variações das brincadeiras. Além disso, as características do espaço físico podem dirigir a outras questões relacionadas também ao amplo conceito de zona lúdica, bem como ao modo de influência adulta na brincadeira infantil.

Em relação ao espaço externo, pesquisas desenvolvidas em dois parques infantis na cidade de Salvador (Bichara, Fiaes, Marques, Brito \& Seixas, 2006) constataram a centralidade dos equipamentos disponíveis e a influência da conformação física desses locais nas brincadeiras. Foi notado, porém, que a estrutura planejada não impede a expressão da criatividade infantil. O que parece acontecer, por parte das crianças, é um uso criativo e inusitado dos equipamentos existentes.

Outro estudo realizado em playgrounds infantis (o mesmo tipo de parque do estudo anterior), na cidade de Amsterdam (Holanda), aponta que o número de frequentadores nesses locais reflete a qualidade do espaço, o número de crianças morando nas proximidades do playground e a disponibilidade de outros espaços de 
brincadeira na mesma vizinhança (Karsten, 2003). A apreciação dos principais resultados encontrados nesses dois estudos de brincadeiras em parques infantis públicos pode, de forma geral, despertar questões possivelmente inter-relacionadas.

A primeira delas refere-se ao tipo de acesso que as crianças têm aos parques, ou seja, se elas dirigem-se ao local e permanecem acompanhadas por adultos ou não. Por exemplo: no caso dos parques de Amsterdam, o acesso das crianças é livre, ou seja, elas podem se dirigir e permanecer sem supervisão nesses locais; o contrário acontece nos parques de Salvador (no Dique do Tororó e no (ampo Grande), onde foi visto que as crianças são levadas e supervisionadas por adultos (Bichara et al., 2006; Karsten, 2003).

É possível perguntar, desse modo, se a localização do parque pode em alguma medida estar associada à frequência de crianças e aos tipos de brincadeiras desenvolvidas por elas, e como a presença de acompanhantes adultos pode interferir diretamente na dinâmica das brincadeiras. Outras questões podem ser elaboradas também no que concerne à forma como as crianças se agrupam para brincar, como elas se relacionam com o espaço físico dos parques, como delimitam os espaços, como o gênero das crianças interfere nesse cenário, entre outras perguntas possíveis que precisariam de maiores investigações.

Quanto aos ambientes internos, Lordelo e Carvalho (2006), em sua pesquisa em diferentes creches, com crianças de dois e três anos de idade, encontraram resultados que sugerem que a atividade lúdica nos primeiros anos de vida é sensível às condições contextuais em que ela ocorre. Ao mesmo tempo, foi vista uma forte estabilidade, indicando tratar-se de um fenômeno sob controle do organismo. Nessa pesquisa, a influência do ambiente pôde ser percebida, e as variações ocorreram principalmente em função de parceiros sociais e dos recursos materiais disponíveis.

Através da estruturação do espaço para brincar, como mostrado acima, é possível reconhecer como os adultos percebem a brincadeira e como procuram orientar e dirigir as atividades lúdicas infantis. Contudo, todas essas pesquisas em espaços internos e externos podem mostrar que a despeito das condições ambientais e dos recursos disponíveis, ou seja, indiferente ao conceito adulto de local adequado ou bem estruturado para a brincadeira, a criança brinca e pode ela mesma apropriar-se do espaço e construir seus próprios significados sobre ele (Rasmussen, 2004). Ela interage ativamente com o contexto e, conforme investigações (Bichara et al., 2006; Sager, Sperb, Roazzi \& Martins, 2003), elas podem adaptar as brincadeiras a qualquer tipo de espaço disponível, determinar papéis, estipular regras e delegar variados usos a objetos, entre outras possibilidades.

Na mesma direção sugerida por Lordelo e Carvalho (2006), as constatações anteriores encontram, portanto, na medida em que apontam para uma orientação central da criança, um modo de funcionamento básico - a brincadeira - e as maneiras como o ambiente circundante pode favorecê-lo. Assim, o arranjo do ambiente na busca pela promoção de espaço satisfatório e da possibilidade de escolha de parceiros parece constituir uma medida simples e, provavelmente, suficiente para permitir à criança engajar-se em atividades para as quais ela dispõe dos necessários recursos e de motivação para autoexpressão e criação.

\section{Discutindo a diversidade e a função da brincadeira}

Nas pesquisas apresentadas aqui sobre a relação entre brincadeira e contexto, encontram-se alguns pontos que precisam ser destacados. Nelas observa-se um amplo número de fatores que podem afetar o modo de brincar das crianças. Nesse ponto, o conceito de zona lúdica indica, de forma ampla, fatores que em alguma medida, isoladamente ou em conjunto, podem influenciar o modo de brincar das crianças. Além disso, falar em zona lúdica implica a consideração das especificidades dos contextos.

Nesse sentido, outro aspecto relevante nas pesquisas anteriormente destacadas é que no ambiente e nos recursos disponibilizados às crianças para a brincadeira estão incluídas as percepções e as expectativas dos adultos acerca dessa atividade, e isso pode também interferir de diferentes formas. Entretanto, o que se percebe também é que as crianças parecem ter um modo próprio de representar o mundo adulto e de construir suas brincadeiras a partir dele. Sobre isso, podemos concluir, como muitos autores (Bichara et al., 2006; Magalhães, Souza \& Carvalho, 2003; Sager et al., 2003) que a criança mostra-se ativa em sua relação com seu contexto. 
Assim, os resultados encontrados nas pesquisas que descrevem brincadeiras em contextos particulares, junto a todas essas reflexões, indicam evidências de que o brincar figura como um comportamento funcional e adaptativo para o próprio período da infância (como sugere a hipótese ontogenética). Isso se justifica na medida em que, para a abordagem evolucionista, entendemos que um comportamento é funcional quando, em termos de história evolutiva, ele tem adicionado sucesso reprodutivo ou de sobrevivência para os indivíduos através das gerações. Ou, além disso, ele tem implicações adaptativas para um determinado período de vida do indivíduo (Pellegrini \& Smith, 1998), como parece ser o caso da brincadeira infantil.

Em termos ontogenéticos, percebemos que a correspondência entre o brincar e um período prolongado de imaturidade não é mero acaso. A brincadeira durante a infância é uma estratégia importante usada para desenvolver os comportamentos que são adaptáveis aos ambientes que as crianças vivem. Assim, as crianças provam seu ambiente e através da brincadeira podem aprender e praticar os comportamentos adaptáveis a esse ambiente em especial. Nas variadas formas de brincar das crianças, pode-se perceber o caráter plástico do comportamento humano desde o período da infância. Dessa maneira, os comportamentos da brincadeira podem representar, na ontogênese, as principais formas através das quais os fenótipos afetam os processos evolucionários subsequentes (Pellegrini et al., 2007). De forma universal, essa observação remete, de fato, à variação do brincar correspondentemente à variação de contextos, na medida em que a adaptação ao contexto torna-se fundamental à sobrevivência do indivíduo. Segundo Bjorklund e Blasi (2005), isso se dá por conta de cada experiência individual, que é diferente, e também por meio de padrões desenvolvimentais variáveis que podem ocorrer no contato do indivíduo com culturas ou contextos específicos.

Num sentido mais amplo, dado o papel fundamental observado na influência do contexto e da cultura na brincadeira, é possível abordar discussões que procurem entender como a cultura se relaciona com a biologia, ou seja, como a diversidade do desenvolvimento humano se relaciona com os aspectos universais dos indivíduos.

Sob essa perspectiva, Greenfield (2002) considera que a relação cultura-biologia ocorre através de prin- cípios complementares - cultura reforçando a biologia, cultura apropriando-se da biologia, cultura e biologia adaptando-se mutuamente para a sobrevivência, cultura selecionando através da biologia e cultura respeitando a biologia. Esses princípios podem ser entendidos em termos de hipótese para o estudo desse relacionamento, mas o que pode ser destacado nesta discussão é que, assim como dito por Keller (2007), a espécie humana é preparada para adquirir, criar e transmitir cultura. E isso é essencialmente o que vemos através do cotidiano da brincadeira infantil, na diversidade produzida pelas crianças.

\section{Referências}

Bichara, I. D., Fiaes, C. S., Marques, R. L., Brito, T., \& Seixas, A. A. C. (2006). Brincadeiras no contexto urbano: um estudo em dois logradouros de Salvador (BA). Boletim Academia Paulista de Psicologia, 26 (2), 39-52.

Bichara, I. D., Lordelo, E. R., Carvalho, A. M. A., \& Otta, E. (2009). Brincar ou brincar: eis a questão, perspectiva da psicologia evolucionista sobre a brincadeira. In M. E. Yamamoto \& E. Otta (Orgs.), Psicologia evolucionista (pp.104-113). Rio de Janeiro: Guanabara-Koogan.

Bjorklund, D. F. (1997). The role of immaturity in human development. Psychological Bulletin, 122 (2), 153-169.

Bjorklund, D. F., \& Blasi, C. H. (2005). Evolutionary developmental psychology. In D. M. Buss (Org.), The handbook of evolutionary psychology (pp.5-67). Hoboken: Wiley.

Carvalho, A. M. A. (1989). Brincar juntos: natureza e função da interação entre crianças. In C. Ades (Org.), Etologia: de animais e de homens (pp.199-210). São Paulo: Edicon.

Carvalho, A. M. A., \& Pedrosa, M. I. (2002). Cultura no grupo de brinquedo. Estudos de Psicologia (Natal), 7 (1), 181-188.

Gosso, Y. (2005). Pexe oxemoarai: brincadeiras infantis entre os índios Parakanã. Tese de doutorado não-publicada, Programa de Pós-Graduação em Psicologia Experimental, Universidade de São Paulo.

Greenfield, P. M. (2002). The mutual definition of culture and biology in development. In H. Keller, Y. H. Poortinga \& A. Shoelmerich (Orgs.), Between culture and biology: perspectives on ontogenetic development (pp.57-76). London: Cambridge University Press.

Johnson, J. E., Christie, J. F., \& Yawkey, T. D. (1999). Play and early childhood development. New York: Longman.

Karsten, L. (2003). Children's use of public space: the gendered world of the playground. Childhood, 10 (4), 457-472.

Keller, H. (2007). Cultures of infancy. London: Lawrence Erlbaum. 
Lordelo, E. R., \& Carvalho, A. M. A. (2006). Padrões de parceria social e brincadeira em ambientes de creches. Psicologia em Estudo (Maringá), 11 (1), 99-108.

Magalhães, C. M. C., Souza, A. R., \& Carvalho, A. M. A. (2003). Piras no riacho doce. In A. M. A. Carvalho, C. M. C. Magalhães, F. A. R. Pontes \& I. D. Bichara (Orgs.), Brincadeira e cultura: viajando pelo Brasilque brinca (pp.77-88). São Paulo: Casa do Psicólogo.

Mekideche, T. (2004). Espaços para crianças na cidade de Argel: um estudo comparativo da apropriação lúdica dos espaços públicos. In E. T. O. Tassara, E. P. Rabinovich \& M. C. Guedes (Orgs.), Psicologia e ambiente (pp.143-167). São Paulo: Educ.

Moraes, M. S., \& Otta, E. (2003). Entre a serra e o mar. In A. M. A. Carvalho, C. M. C. Magalhães, F. A. R. Pontes \& I. D. Bichara (Orgs.), Brincadeira ecultura: viajando pelo Brasilque brinca (pp.127-156). São Paulo: Casa do Psicólogo.

Pellegrini, A. D., \& Smith, P. K. (1998). The development of play during childhood: forms and possible functions. Child Psychology and Psychiatry Review, 3 (2), 51-57.

Pellegrini, A. D., Dupius, D., \& Smith, P. K. (2007). Play in evolution and development. Developmental Review, 27, 261-276.
Pontes, F. A. R., \& Magalhães, C. M. C. (2003). A transmissão da cultura da brincadeira: algumas possibilidades de investigação. Psicologia: Reflexão e Crítica, 16 (1), 117-124.

Rasmussem, K. (2004). Places for children: children's places. Childhood, 11 (2), 155-173.

Sager, F., Sperb, T. M., Roazzi, A., \& Martins, F. M. (2003). Avaliação da interação de crianças em pátios de escolas infantis: uma abordagem da psicologia ambiental. Psicologia: Reflexão e Critica, 16 (1), 203-215.

Santos, A. K. (2005). Um estudo sobre brincadeira e contexto no agreste sergipano. Dissertação de mestrado não-publicada, Programa de Pós-Graduação em Psicologia, Universidade Federal da Bahia, Salvador.

Seixas, A. A. C. (2007). Brincando na llha dos Frades. Dissertação de mestrado não-publicada, Programa de Pós-Graduação em Psicologia, Universidade Federal da Bahia, Salvador.

Recebido em: 22/6/2009

Versão final reapresentada em: 12/11/2010

Aprovado em: 11/3/2011 